\title{
The effect of hand position and pattern motion on temporal order judgments
}

\author{
JAMES C. CRAIG \\ Indiana University, Bloomington, Indiana
}

\begin{abstract}
Subjects made temporal order judgments (TOJs) of tactile stimuli presented to the fingerpads. The subjects judged which one of two locations had been stimulated first. The tactile stimuli were patterns that simulated movement across the fingerpads. Although irrelevant to the task, the direction of movement of the patterns biased the TOJs. If the pattern at one location moved in the direction of the second location, the subjects tended to judge the first location as leading the second location. If the pattern moved in the opposite direction, that location was judged as trailing. In a series of experiments, the effect of the spatial position of the hands and fingers on TOJs and the perception of the direction of pattern movement were examined. Changing the position of the hands so that the patterns no longer moved directly toward each other reduced or eliminated the effect of motion on TOJs. In a variation of Aristotle's illusion, the moving patterns were presented to crossed and uncrossed fingers. The results indicated that, contrary to Aristotle's illusion, the subjects processed the moving patterns relative to an environmental framework, rather than to the local direction of motion on the fingerpads. Presenting the patterns to crossed hands produced results similar to those obtained with crossed fingers: The subjects processed the patterns according to an environmental framework.
\end{abstract}

In a recent study of temporal order judgments (TOJs), subjects judged which one of two sites on the hand was stimulated first (Craig \& Busey, 2003). The stimuli were moving patterns generated on two tactile arrays. At brief stimulus onset asynchronies (SOAs), the subjects based their TOJs, to a large extent, on the direction of motion across the fingers, even though the motion was irrelevant to the TOJ. For example, when the patterns were presented to the index and middle fingerpads on the pronated left hand and the patterns moved from right to left, the subjects showed a strong bias to respond that the index finger had received the first stimulus. If the patterns moved from left to right, the subjects showed a strong bias to respond that the middle finger had received the first stimulus. The condition was referred to as consistent when, for example, the movement at the two sites was from right to left and the index finger site (the site to the right) led the site to the left. The term consistent was used because the direction of motion across the two fingers was consistent with the type of stimulation received in haptic exploration. If the left hand is moved to the right across a surface, the surface, relative to the fingerpad, moves from right to left, and surface features are encountered by the index fingerpad before they are encountered by the middle finger. Inconsistent movement

This research was supported by National Institutes of Health Grant DC00095, National Institute on Deafness and Other Communication Disorders. I thank Roger Rhodes for his assistance in these experiments and Carol Rhodes for the illustrations. Correspondence concerning this article should be addressed to J. C. Craig, Department of Psychology, Indiana University, Bloomington, IN 47405 (e-mail: craigj@indiana.edu). is generated by reversing the direction of motion across the fingerpads (making it left to right) while the index fingerpad still receives the first pattern (Craig \& Busey, 2003).

At SOAs of less than $50 \mathrm{msec}$, consistent conditions led to correct responding on as many as $80 \%$ of the trials, whereas inconsistent conditions led to correct responding on as few as $20 \%$ of the trials. The use of moving, as compared with static, patterns did not lower overall performance in TOJs - that is, overall correct performance was the same for the moving patterns as for the static patterns. Because the moving patterns did not alter sensitivity, the effect of movement appears to be one of biasing the subjects' responses (Craig \& Busey, 2003).

In the earlier study, testing initially involved adjacent fingers on the same hand. On the basis of the results from these tests, it was thought that moving stimuli affected TOJs because of the close coupling of the movement of objects across the hand and the temporal order in which features are received at the skin's surface. This explanation became less plausible as additional conditions were tested. Moving patterns produced similar biases in TOJs when the sites of stimulation were fingers on opposite hands (bilateral). Moreover the bias was the same whether the two hands were placed close together or far apart $(80 \mathrm{~cm})$. When two hands are placed $80 \mathrm{~cm}$ apart, it is unlikely that there is a very close coupling of the temporal order of features arriving at the two hands and the direction of movement. What all the conditions had in common was that the leading pattern had to be moving toward the other location or the trailing pattern had to be moving away from the other location; however, no con- 
ditions were examined in which the motion was not directly toward or away from the sites of stimulation. In the present study, the term leading toward is used to describe trials in which the temporally leading pattern is moving in the direction of the site of the temporally trailing pattern, similar to the consistent condition. Leading away refers to trials in which the leading pattern is moving away from the other site of stimulation, similar to the inconsistent condition. On leading-away trials, it is the trailing stimulus that moves in the direction of the leading stimulus.

One of the aims of the present study was to examine additional conditions that might affect the TOJ bias. In all of the conditions previously tested, the two tactile displays were placed parallel to each other and horizontally in front of the subjects. The patterns moved in the lateralmedial direction relative to the body. In the present study, the effects of altering the spatial positions of the displays on TOJs were investigated. To examine further the explanation that the coupling of temporal order and movement across the skin surface accounts for the TOJ bias, we presented the moving patterns to the fingers and hands in positions in which such coupling was unlikely. For example, the subjects made TOJs of moving patterns presented to two fingers crossed over one another (Experiment 2) and to two hands crossed over one another (Experiment 3). The subjects were very unlikely to have engaged in extensive haptic exploration with their hands or fingers crossed.

The second major aim of the study was to explore the spatial frame used by subjects in processing moving patterns. The hand is a unique sensory organ. It can be used both to manipulate objects and to gain perceptual information. In view of the fact that the hand has a number of degrees of freedom, the local pattern of sensory information received at a particular site on the hand often has to be interpreted in light of the position of the hand in space. Some studies have shown that the perception of the pattern of local motion on the hand or other skin surfaces depends on the position of the stimulated part in space (Oldfield \& Phillips, 1983; Parsons \& Shimojo, 1987; Sekiyama, 1991). For example, the letter "b" drawn on the palm facing the observer will generally be perceived as the letter "b," but when the same local pattern is drawn on the palm facing away from the observer, the letter "d" is perceived. In a study of selective attention, target and distractor stimuli were presented to fingertips on opposite hands. The amount of interference declined as the hands were moved farther apart, indicating that the task was sensitive to an external spatial frame of reference, rather than to a somatotopic frame of reference (Driver \& Grossenbacher, 1996). Under some circumstances, however, subjects' spatial judgments remain unchanged as the position of the stimulated sites is altered (Benedetti, 1988; Rieser \& Pick, 1976). How and under what circumstances spatial perception is altered depends on a number of variables. One of the difficulties in studying the effects of spatial position on pattern perception is that subjects may attempt to adopt the perspec- tive of the experimenter and respond with the pattern that they think the experimenter intended to present.

A previous study (Rinker \& Craig, 1994) in which moving patterns were used attempted to avoid the problem of the results being biased because of task requirements. In that study, subjects attempted to identify the direction of motion of a target pattern presented to the thumb. A second, irrelevant moving pattern was presented to the index finger. When the two patterns were moving in opposite directions, the irrelevant pattern interfered with the identification of the target pattern. This testing was done with the two displays parallel to each other in the transverse plane (horizontally in front of the subject). The two displays were then oriented vertically. The subjects gripped the two displays between the thumb and the index finger. The task was the same: attend selectively to the thumb and report the direction of motion. The change in orientation of the displays altered the pattern of interference from the irrelevant stimulus. The irrelevant stimulus that had produced no interference when the two displays were horizontal now resulted in interference, and vice versa. In the present study, the bias in TOJs seen with moving stimuli was used as a way of examining the perception of moving stimuli.

As was noted, one of the aims of the present study was to examine how the spatial orientation of the hands would affect the perception of the direction of motion and the TOJ bias. If the local pattern of motion is kept the same at the two sites, does the bias disappear as the hands are moved and the direction of motion is no longer toward one of the sites of stimulation? Does changing the position of the hands in space alter TOJs? Do subjects show evidence of perceptual constancy in processing the moving patterns?

These questions were examined in three experiments. In Experiment 1, the displays and the index fingers contacting the displays were changed from being parallel to one another $\left(0^{\circ}\right)$ to having the displays form a $90^{\circ}$ angle and a $180^{\circ}$ angle. In Experiment 2, the moving patterns were presented to two fingers on the same hand when the fingers were both uncrossed and crossed. The intent was to replicate conditions in which subjects fail to show perceptual constancy and greatly mislocalize stimuli spatially (Benedetti, 1988). In Experiment 3, the patterns were presented to uncrossed and crossed hands. The latter condition has been shown to interfere greatly with TOJs of static stimuli (Shore, Spry, \& Spence, 2002; Yamamoto \& Kitazawa, 2001a, 2001b).

There are several advantages to using TOJs to examine the interaction between tactile motion and limb position. First, the effects of TOJs are relatively large and reliable. Second, subjects are not asked to judge the position of the fingers and hands in space or to make any judgments of motion. In fact, in these studies, neither the position of the limbs nor the direction of motion is relevant to the TOJs. Subjects do not have to attempt to interpret instructions about whether the direction of motion should be judged solely on the basis of the local pattern of motion on the fingerpads (somatotopic frame- 
work) or relative to the position of the hands in space (environmentally based framework). In haptic exploration or in manipulating an object, complex patterns of motion across the fingerpads are generated, but attention is rarely focused on these patterns per se. Rather, the observer is trying to judge the shape of an object, its position in space, or whether some parts can be moved relative to others. Observers are generally more concerned with the environmental, rather than the somatotopic, frame of reference. Third, the question has been raised in studies of haptic and tactile spatial judgments of whether the judgments are verbally mediated (Rieser \& Pick, 1976). Inasmuch as subjects are making no judgments of motion, the effects of pattern motion on TOJs are unlikely to be mediated verbally.

\section{EXPERIMENT 1}

In Experiment 1, the effect on the TOJ bias of altering the position of the hands in space and the directions of motion in an environmental frame were examined. Figure 1 shows the position of the left and right index fingers at various positions. Arrows indicate the direction of motion on the fingers. The previous study had examined only the position marked " 0 "." At $90^{\circ}$ the motion was no longer moving directly from one display to the other. If the motions at the two displays were extended toward each other, their trajectories would intersect at a $90^{\circ}$ angle midway between the two displays and about $5 \mathrm{~cm}$ below a line segment connecting the two displays. At $180^{\circ}$, the motions at the two sites were parallel to each other. If the TOJ bias depends on the direction of motion in an environmental framework and if perception of the direction of motion changes with hand position, the size of the TOJ bias might decrease, perhaps to zero, as the angle between the two hands changed from $0^{\circ}$ to $90^{\circ}$ to $180^{\circ}$. The question was whether there would be any change in TOJ performance as a function of the placement of the hands.

\section{Method}

Subjects. The subjects in all the experiments were undergraduate students at Indiana University. Generally, the subjects had participated in several tactile experiments. They were selected for participation in each experiment as scheduling permitted. They received an hourly wage for their participation. Four women and 2 men were tested.

Apparatus. Two tactile displays generated the stimuli. Each display consisted of 144 tactors arranged in a 6-column $\times 24$-row array. The tactors were activated with square waves, $230 \mathrm{pps}$. The two displays were interfaced with a PC. The arrays were similar to those used in the Optacon, a reading aid for the blind (Bliss, Katcher, Rogers, \& Shepard, 1970). The arrays fit against the distal portion of the fingerpads. The patterns covered an area $17 \times 11 \mathrm{~mm}$.

Procedure. The subject's task was to indicate which of the two sites of stimulation received the stimulus first. The subjects placed the index finger of the left hand on one display and the index finger of the right hand on the other display. The moving patterns were generated by activating pairs of columns sequentially on the display. A representation of the patterns is shown in Figure 2. Each pair of columns was activated for $13 \mathrm{msec}$. The SOA between columns was $13 \mathrm{msec}$. The pair of columns occupied five different locations on the display for a total duration of $65 \mathrm{msec}$ and covered
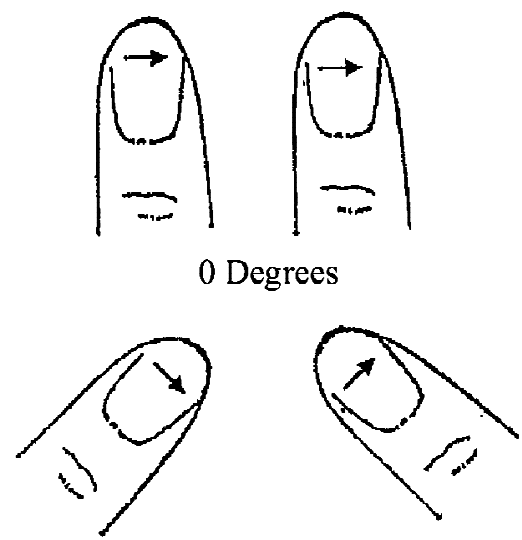

90 Degrees
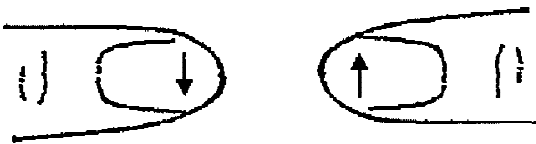

180 Degrees

Figure 1. A representation of the position of the index fingers in Experiment 1: $0^{\circ}, 90^{\circ}$, and $180^{\circ}$. The arrows indicate the direction of motion of the patterns across the fingerpads for one type of trial. In a second type of trial, the motion at both fingerpads was in the opposite direction.

a distance of $11 \mathrm{~mm}$. The voltage to each tactor was set so as to produce a moderate stimulus intensity. The resulting sensation was one of a light mechanical stimulus moving across the fingerpad.

The subjects initiated each trial with a foot pedal. Following the presentation of the pair of stimuli, the subjects responded by means of two foot pedals to indicate which of the two stimuli they judged to have been presented first. The foot pedals were placed side by side. The subjects typically used the left foot to depress the left pedal and the right foot to depress the right pedal. No trial-by-trial feedback was provided. The subjects were instructed that the direction of motion was irrelevant to the TOJ.

The patterns presented to the two fingers moved in the same direction on both fingers. The direction of motion and the temporal order were determined randomly on a trial-by-trial basis. The direction of motion was uncorrelated with the temporal order. Three positions of the arrays (and the positions of the hands and fingers) were tested in Experiment 1 (Figure 1). The distance between the two arrays for these three positions was $10 \mathrm{~cm}$. A single position was tested each session. The directions of movement of the patterns across the fingerpads were the same for the three positions. The order of testing of the positions was random with the constraint that each position was tested once before being tested again.

Six SOAs were tested; 400, 200, 100, 52, 26, and 13 msec. Each block of 50 trials tested a different SOA. In each experimental session, the six SOAs were tested in order from longest to shortest. We began with the 400-msec SOA. At this SOA, there was no bias, and the subjects were generally correct. This provided a clear demonstration of the nature of the task for the subjects and showed them that the direction of the pattern movement was irrelevant to the TOJ The subjects were generally able to complete two sets of six SOAs each (a total of 600 trials) in a single session. To eliminate auditory cues from the two displays, the subjects wore earplugs and earphones through which white noise was presented. For each of the three conditions $\left(0^{\circ}, 90^{\circ}\right.$, and $\left.180^{\circ}\right)$, each of the 6 subjects completed four sets of the six SOAs, a total of 7,200 trials at each of the three positions. 


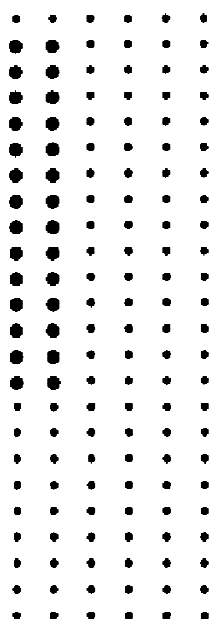

Frame 1

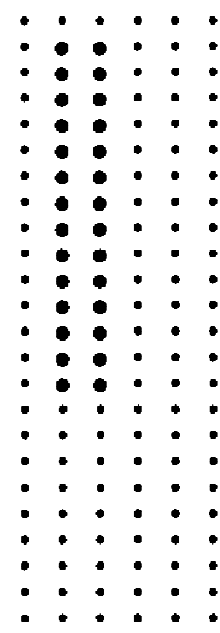

Frame 2

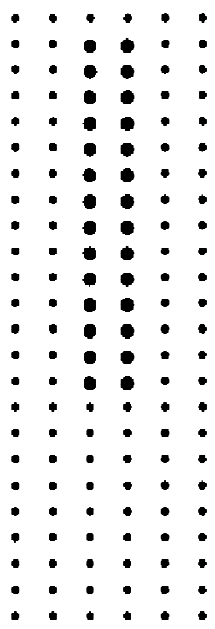

Frame 3

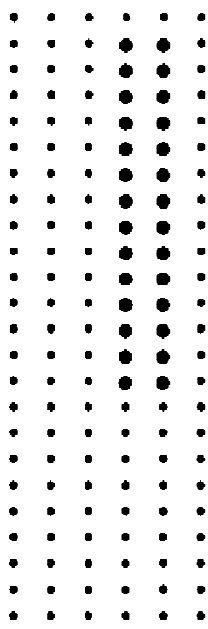

Frame 4

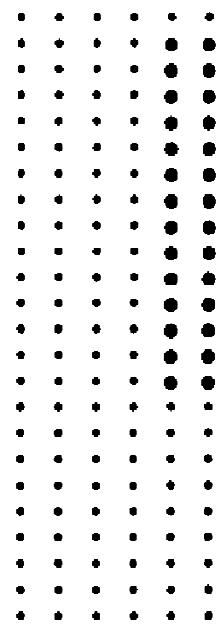

Frame 5

Figure 2. A representation of the tactile display and moving patterns. Each panel represents one frame of the tactile pattern moving from left to right.

\section{Results and Discussion}

The results for the three positions of the arrays are shown in Figure 3. The percentage of correct responses is plotted as a function of SOA. The top panel shows the results when the two displays were at $0^{\circ}$. Three functions are presented. The top function, marked "leading toward," refers to trials on which the pattern at the temporally leading site was moving toward the site at which the trailing pattern was presented. As was noted, in the previous study (Craig \& Busey, 2003), this condition was referred to as consistent movement. The lower function, "leading away," refers to trials on which the temporally leading stimulus was moving away from the site of the trailing stimulus, previously referred to as inconsistent movement. The middle function is the mean of the upper and lower functions, approximately equal to the overall level of correct performance. These results were similar to the results obtained in the previous study: There was a bias to respond that the pattern that was moving toward the second location was the temporally leading pattern. When the pattern moving toward the second location was, in fact, the leading pattern, the subjects tended to be correct. When the pattern moving away from the second location was the leading pattern, the subjects tended to be incorrect (lower function). An analysis of variance (ANOVA) showed a significant effect of trial type [leading toward vs. leading away; $F(1,5)=24.37, p<.01$ ]

The middle panel presents the results from the $90^{\circ}$ condition. Although the direction of motion at the two sites was no longer directly toward each other, there was still an effect of the direction of pattern motion and a bias favoring the leading-toward trials $[F(1,5)=13.78, p<$ $.05]$. In the lower panel, the $180^{\circ}$ condition, the patterns were now moving parallel to each other in an environmental frame, rather than toward one another; however, the same labels for the functions were retained-that is, "leading toward" referred to the same frame of reference as in the other panels (local pattern of motion or somatotopic). The same labels for the local directions of motion were retained to show more clearly the effect of the positions of the hand and, specifically, that pattern motion no longer affected TOJs $[F(1,5)=0.88, p=.39]$. These results indicate that the TOJ bias depended, as was expected, on the spatial position of the sites of stimulation: As the position changed, so did the TOJ bias.

To see more clearly the effect that hand position and the direction of motion (environmental frame) has on TOJs, we calculated the difference in performance at each SOA between the leading-toward and the leadingaway trial types. These differences are plotted in Figure 4 and show that the magnitude of the TOJ bias declined with the angle between the two displays. An ANOVA showed a significant effect of position of the arrays $[F(2,10)=10.17, p<.01]$. An ANOVA performed on just the $0^{\circ}$ and $90^{\circ}$ positions showed no significant difference in the size of the TOJ bias between these two positions $[F(1,5)=3.98, p=.10]$; however, a separate analysis of the three briefest SOAs, where the TOJ bias is most evident, did show a significant effect of position $[F(1,5)=7.57, p<.05]$. This result suggests that the effect of altering the angle between the arrays did not result in an all-or-none effect but, rather, that the TOJ bias was a continuous function of the relative direction of motion across the two hands.

\section{EXPERIMENT 2}

In Experiment 2, subjects made TOJs of moving patterns presented to two fingers on the same hand. The issue was again whether the subjects would correctly perceive the direction of motion (environmental frame), as reflected in TOJs, when the positions of the sensory surfaces were altered spatially. In Experiment 2, the spatial positions were altered by crossing the fingers. The 

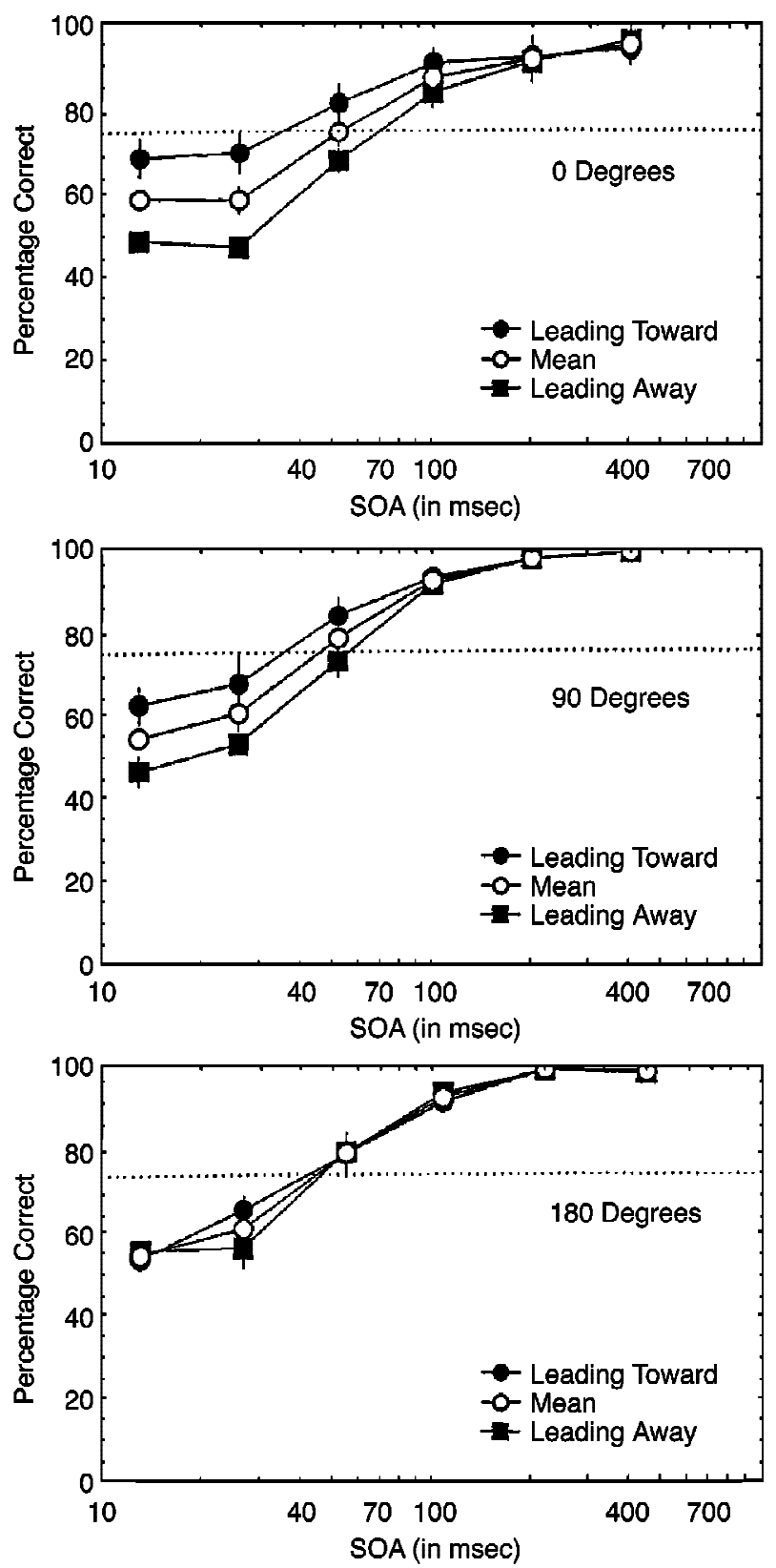

Figure 3. Percent correct in judging temporal order as a function of the stimulus onset asynchrony (SOA) in milliseconds. The three panels present the data from the three positions of the hands in Experiment 1: $0^{\circ}, 90^{\circ}$, and $180^{\circ}$. In the results from the $0^{\circ}$ condition, the top function represents those trials on which the leading pattern was moving toward the other site of stimulation. The bottom function represents those trials on which the leading pattern was moving away from the other site of stimulation. The "mean" function represents overall percent correct, the mean of the upper and lower functions. The $90^{\circ}$ results are labeled in the same way as the $0^{\circ}$ results, as are the $180^{\circ}$ results. For the $180^{\circ}$ results, the same local patterns of motion (somatotopic frame) on the fingerpads are labeled as they are in the other results, although the patterns are now moving parallel to one another (environmental frame). Error bars represent \pm 1 standard error of the mean. reason for being particularly interested in the effect of crossing fingers comes from a series of experiments by Benedetti (1985, 1986a, 1986b, 1988, 1991) and is related to Aristotle's illusion. In Aristotle's illusion, two fingers on the same hand are crossed, such as the middle and the index fingers, and a rod is placed between the two fingertips. The observer typically perceives two rods. Benedetti examined and quantified some additional perceptual effects of crossing the fingers. In Benedetti's (1988) finding most relevant for the present experiment, subjects crossed finger D3 over D4. The subjects were touched simultaneously on the tips of the two fingers and were asked to localize the two points of stimulation relative to one another. When the third finger (right hand) was placed above the fourth finger, the subjects accurately perceived the stimulus presented to the third finger as being directly above the stimulus presented to the fourth finger. The subjects then moved their fingers from this position and crossed $\mathrm{D} 3$ over $\mathrm{D} 4$ so that $\mathrm{D} 3$ was now to the right of D4. The unexpected finding was that the subjects were almost completely unable to take the crossed position of the fingers into account in localizing the points. The subjects continued to localize D4 directly above D3, not in its new position to the right of D3. In other words, the subjects did not take into account the fact that the fingers were now crossed (Benedetti, 1988). Benedetti pointed this out as an example of a failure of perceptual constancy.

The question in Experiment 2 was whether subjects with crossed fingers would perceive the direction of motion as though their fingers remained uncrossed. Figure 5 shows representations of the patterns with fingers uncrossed and crossed. Suppose that the two patterns were moving from left to right and the middle finger (uncrossed condition) received the stimulus first (panel A). Previous results indicate that in this leading-toward condition, subjects are very likely to correctly select the middle finger as leading the index finger. Suppose that the identical patterns, same directions of motion, are generated on the displays but now the fingers are crossed (Figure 5, panel B). The index finger would now be receiving the pattern first. If subjects correctly took account of the fact that the patterns were being processed by crossed fingers, they should tend to respond that the index finger was the leading site. This result would be evidence for perceptual constancy. If, however, as Benedetti's (1988) results predict, subjects fail to perceive that the middle finger is now to the right of the index finger, the TOJ bias could disappear, or subjects could respond that the middle finger trails the index finger (somatotopic frame). Such results would show a lack of perceptual constancy.

\section{Method}

Subjects. Six women and 2 men were tested.

Procedure. The procedure was similar to that used in Experiment 1 . The subjects' task was to respond with the location that re- 


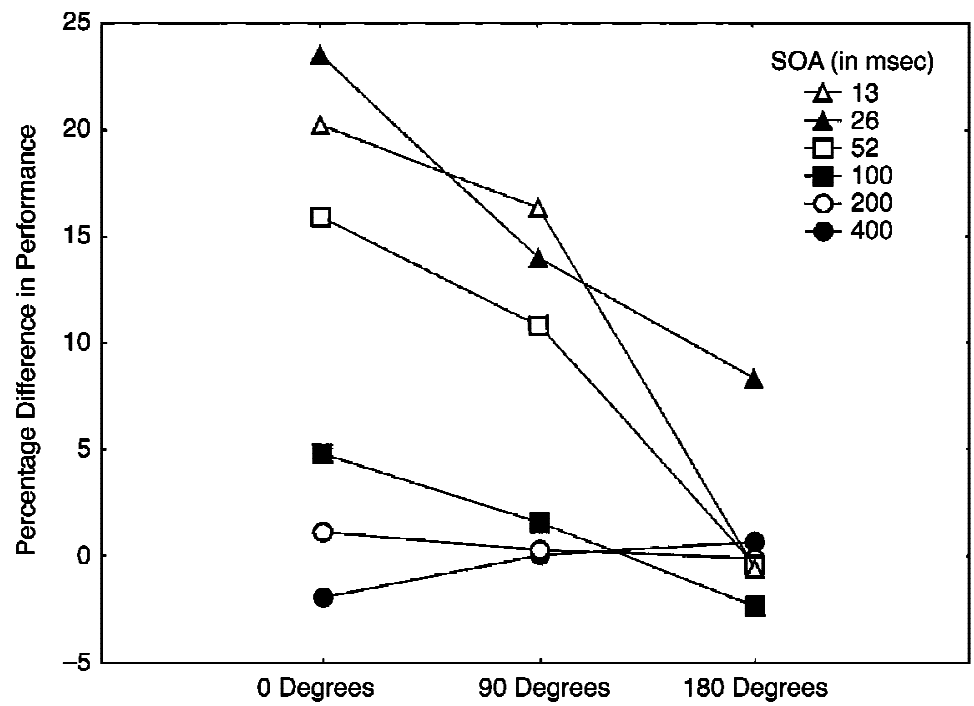

Figure 4. The difference in percent correct between the leading-toward and leading-away functions in Figure 3-that is, the size of the TOJ bias, plotted as a function of the angle between the positions of the fingers. The parameter is the stimulus onset asynchrony (SOA), in milliseconds, between the two patterns.

ceived the stimulus first. The index and middle fingers of the left hand were the sites of stimulation. A single display was used. In order for the two fingers to contact the array, the array was rotated $90^{\circ}$. In this configuration, what had been rows of the display became columns, and vice versa. The stimuli consisted of a pattern three tactors in width and six tactors in height. The stimuli moved laterally across the fingerpads, with each element of the pattern occupying a position in the proximal/distal orientation for $13 \mathrm{msec}$. There were $13 \mathrm{msec}$ between onsets of successive positions across the fingerpad. The patterns occupied eight positions along the array, a distance of $8.8 \mathrm{~mm}$. The total duration for each pattern was $104 \mathrm{msec}$. In the testing sessions, the blocks of trials alternated between crossed and uncrossed conditions. In the first block in each session, the fingers were uncrossed. Six SOAs-400, 200, 100, 52, 26, and $13 \mathrm{msec}$ - were tested in each session. Blocks consisted of 25 trials. Eight subjects were tested for four blocks for each of the six SOAs, a total of 4,800 trials for the uncrossed condition and 4,800 trials for the crossed condition.

\section{Results and Discussion}

The results with the uncrossed fingers are shown in Figure 6. The functions are labeled as in Figure 3. The data from the two directions of motion, left and right, are presented in separate panels to make it easier to compare the results from the uncrossed condition with the results from the crossed condition. The top panel shows the results when the two patterns were moving to the right. As was expected, when the fingers were uncrossed and when the pattern at the leading site was moving in the direction of the second site, the subjects were very likely to respond correctly (leading-toward trials). When the pattern at the leading site was moving in a direction away from the second site, the subjects were, at brief SOAs, very unlikely to respond correctly (leading-away trials). These results are similar to those seen in previous measure- ments. For both the moving-right and the moving-left conditions, there was a significant effect of trial type $[F(1,7)=103.82, p<.001$, and $F(1,7)=92.38, p<.001$, respectively].

The results with the crossed fingers are shown in Figure 7. To help visualize the effects of motion, the results from the two directions of motion are presented separately, as they were in Figure 6. The top panel shows the results when the two patterns were moving to the right. In discussing these results, the terms left and right refer to positions of the patterns on the array (environmental frame), not to the fingers (either crossed or uncrossed). The top function, marked "leading toward," refers to those trials on which the pattern on the left side of the array was presented first. These results show that when both patterns were moving from left to right, there was a strong bias to respond that the site on the left (in this case, the index finger) was leading. When the site on the left received the stimulus first, the subjects were correct (top function). In this crossed-finger condition, the left side of the array was contacted by the left index finger (Figure 5). Thus, the leading pattern was moving toward the site of the trailing pattern (leading toward) - that is, index toward middle finger. Moreover, the results were the same whether the middle finger (uncrossed condition) or the index finger (crossed condition) was contacting the left side of the array. When the site on the left was trailing, the subjects were incorrect (bottom function).

The pattern of results in Figure 7 (top panel) is predicted by perceptual constancy. If the fact that the fingers are crossed had not been taken into account and the subjects had responded as though their fingers were un- 

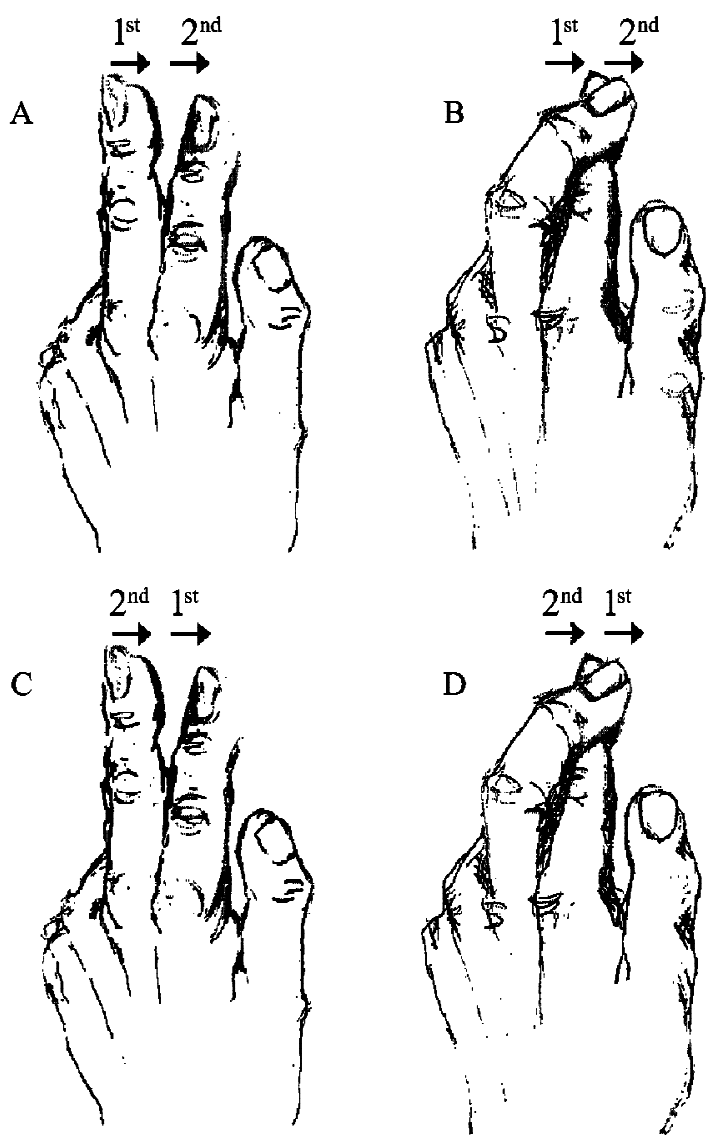

Figure 5. A representation of the direction of motion (to the right) and temporal order (1st and 2 nd) for patterns presented to fingers uncrossed and crossed. Panels $A$ and $B$ are leadingtoward trial types, as defined by an environmental frame. Panels $C$ and $D$ are leading-away trial types, as defined by an environmental frame. As defined by the local pattern of motion (somatotopic frame), panel $B$ is a leading-away trial type, and panel $D$ is a leading-toward trial type.

crossed, the bias would have been in the opposite direction: The subjects would have responded that the index finger trailed the middle finger, because with the same patterns presented to uncrossed fingers, the direction of movement on the index finger would be moving away from the middle finger. An ANOVA showed a significant effect of trial type $[F(1,7)=20.21, p<.01]$.

The bottom panel of Figure 7 shows the results with crossed fingers when the patterns were moving to the left. These results mirror those in the top panel and show the same bias: Patterns moving toward the other location were selected as the leading pattern. In this condition with both patterns moving from right to left, there was a strong bias to respond as though the pattern on the right was the leading pattern. An ANOVA showed a significant effect of trial type $[F(1,7)=59.38, p<.01]$. As with the patterns moving to the right (Figure 7, top panel), the subjects' performance indicates that when moving patterns are processed, crossing the fingers does not lead to a misperception of the direction of motion as referenced externally.

Overall sensitivity in the crossed condition appears to be somewhat poorer than that in the uncrossed condition. An ANOVA showed that there was a significant difference in overall performance between the crossed and the uncrossed conditions $[F(1,7)=13.21, p<.01]$. This difference is also reflected in the thresholds, $75 \%$ correct, which were calculated by linear interpolation. In the uncrossed condition, the threshold was $128 \mathrm{msec}$, and in the crossed condition, it was $175 \mathrm{msec}$. Sensitivity in the uncrossed condition also appears to be rather poor; however, the threshold, $128 \mathrm{msec}$, is close to the threshold obtained in the previous study under similar conditions. That threshold was $101 \mathrm{msec}$ (Craig \& Busey, 2003).

\section{EXPERIMENT 3}

Several recent studies compared TOJs for stimuli presented to crossed and uncrossed hands (Shore et al., 2002; Yamamoto \& Kitazawa, 2001a, 2001b). Subjects judged whether a tactile stimulus had been delivered to
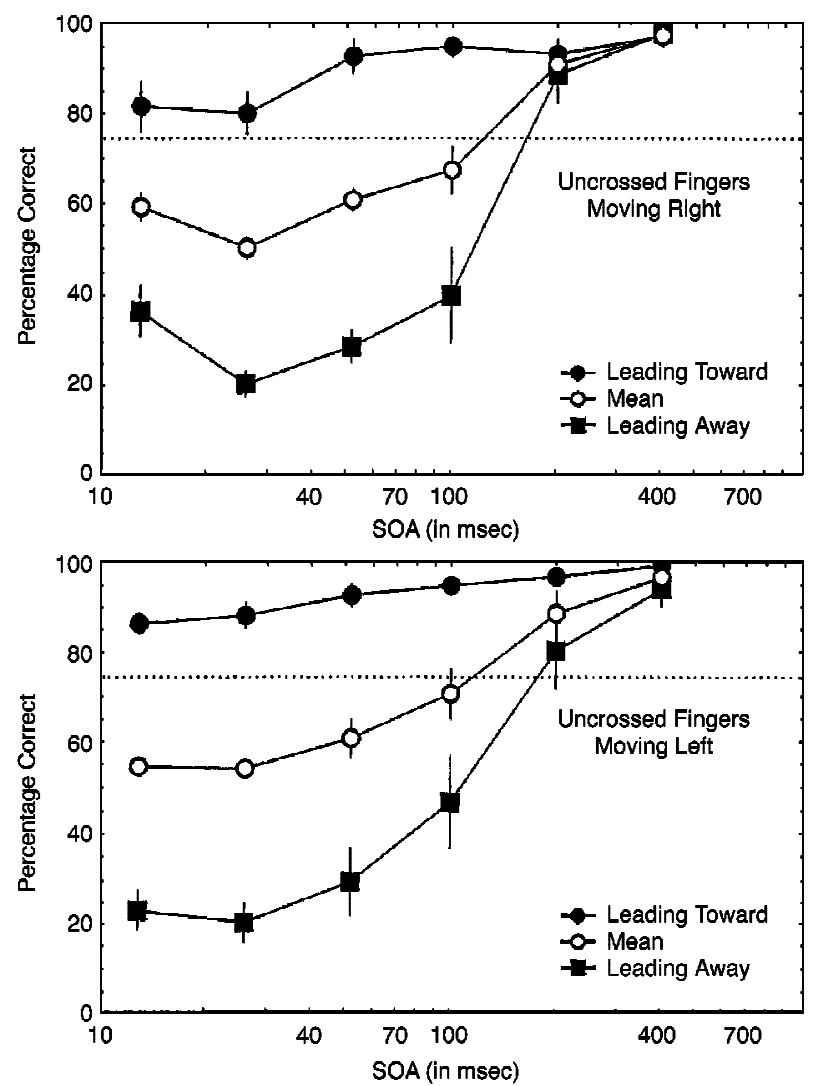

Figure 6. Performance in judging temporal order. Patterns were presented to two fingers, uncrossed. The functions are labeled as in Figure 3. The top panel presents the results when the two patterns were moving to the right. The bottom panel presents the results when the two patterns were moving to the left. Error bars represent \pm 1 standard error of the mean. 

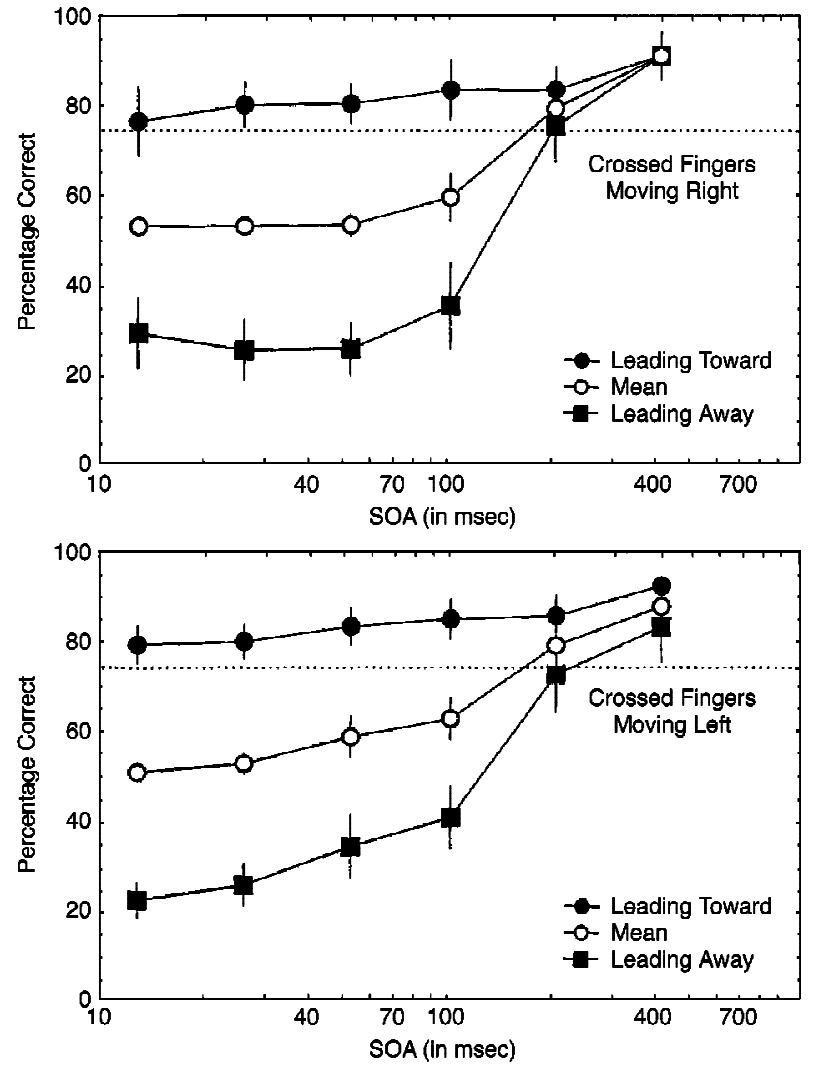

Figure 7. Performance in judging temporal order. Patterns are presented to two fingers, crossed. The top panel presents the results when the two patterns are moving to the right. The bottom panel presents the results when the two patterns are moving to the left. The functions are labeled using an environmental frame, rather than a somatotopic frame (Figure 5). Error bars represent \pm 1 standard error of the mean.

the right hand before the left hand or vice versa. Crossing the hands resulted in large increases in the threshold for temporal order, as compared with uncrossed conditions. In one study, with uncrossed hands, subjects could correctly report the temporal order on more than $80 \%$ of the trials at temporal intervals of approximately $70 \mathrm{msec}$ (Yamamoto \& Kitazawa, 2001a). With the hands crossed, similar levels of performance were not achieved until the intervals were greater than 300 msec. In Shore et al.'s study, the thresholds in the crossed condition were, in some conditions, more than 3 and one half times larger than the thresholds in the uncrossed condition: $34 \mathrm{msec}$, as compared with $124 \mathrm{msec}$. In addition, Shore et al. showed that the crossed-hand deficit was evident even with experienced subjects. In these studies, the tactile signals were brief, mechanical stimuli-that is, static, rather than moving, stimuli.

In Experiment 3, TOJs were obtained with moving stimuli with hands crossed and uncrossed. The first aim of Experiment 3 was to see whether crossed hands would perturb TOJs when the stimuli were moving patterns. As compared with static patterns, might moving patterns overcome the effects of crossing the hands and produce sensitivity similar to that seen in Experiment 1? The second aim was to see whether subjects use a somatotopic frame or an environmental frame when the hands are crossed. One of the conclusions from Yamamoto and Kitazawa's (2001a) study was that the relatively poor sensitivity they observed was the result of subjects' having to adjust for the crossed-hand position. The authors suggest that subjects assume that their hands are uncrossed and that to make the TOJ remapping is necessary and time consuming. If subjects are assuming that their hands are uncrossed when making TOJs, this should be evident in the effect of the direction of movement on their judgments. Such an effect would be similar to Benedetti's (1988) results with crossed fingers and contrary to the results of Experiment 2. In short, the bias observed with crossed fingers should be reversed.

\section{Method}

Subjects. Five women and 3 men were tested.

Procedure. Preliminary testing indicated that crossing the hands did indeed result in poorer performance, as predicted by earlier studies. Thus, it was necessary to test longer SOAs than in the previous experiments. Specif ically, SOAs of 1,000, 500, 200, 100, 52 , and $26 \mathrm{msec}$ were tested in 50-trial blocks. The same patterns as those used in Experiment 1 were used in Experiment 3. As in Experiments 1 and 2, the subjects were tested in order from the longest to the shortest SOAs within a testing session. The subjects were tested with hands crossed, right hand crossed over the left hand, and with arms uncrossed. Sessions alternated between crossed and uncrossed hands. The subjects were tested for eight sessions, four crossed and four uncrossed. The patterns were presented to the index fingers of the two hands.

\section{Results and Discussion}

The results are presented in Figure 8 and are labeled as in Figure 3. The top panel shows the results with hands uncrossed, and the bottom panel shows the results with hands crossed. In both conditions, the direction of motion affected performance. There was a significant effect of trial type in both the uncrossed $[F(1,7)=13.36, p<$ $.01]$ and the crossed conditions $[F(1,7)=7.87, p<.05]$. The uncrossed-hands condition was, in effect, a replication of the $0^{\circ}$ condition in Experiment 1, and the results were similar.

The focus of Experiment 3 was on the crossed condition. Consistent with the terminology used in Experiment 2, the descriptions of the movement of the patternsmoving toward and moving away - refer to the motion in an environmental framework. When the patterns were moving from left to right, the subjects were likely to respond that the site of stimulation on the left received the stimulus first. If the leading site was on the left, the subjects tended to be correct. If the leading site was on the right, the subjects tended to be incorrect. The fact that the hands were crossed did not affect the direction of the bias. These results do not support the conclusion from Yamamoto and Kitazawa's (2001a) study that the somatosensory system has a default option that assumes 

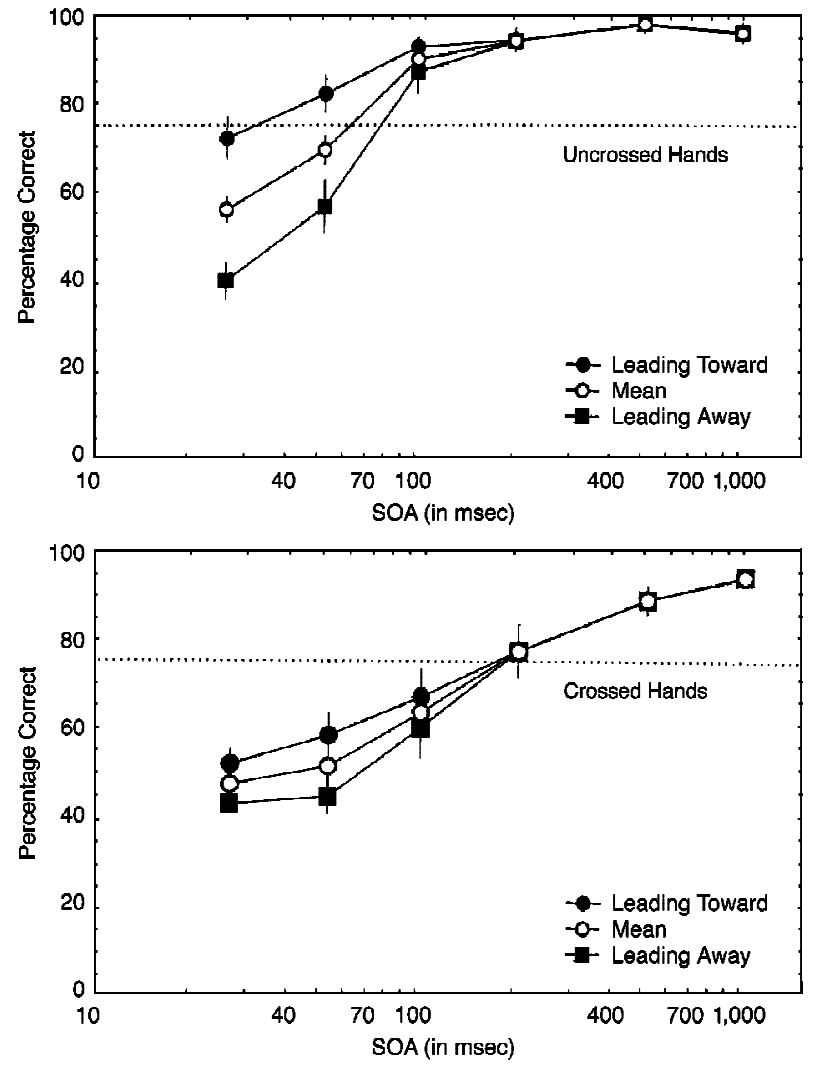

Figure 8. Performance in judging temporal order. Patterns were presented to two hands. The top panel presents the results when the hands were uncrossed. The bottom panel presents the results when the hands were crossed. The functions are labeled using an environmental frame, rather than a somatotopic frame. Error bars represent \pm 1 standard error of the mean.

that the hands are uncrossed. As with the crossed fingers, the subjects' responses indicate that they were aware of the effect of crossing their hands and were using an environmental frame.

Crossing the hands had a clear effect on sensitivity. There was a significant difference between the mean function with uncrossed hands and the mean function with crossed hands $[F(1,7)=20.13, p<.005]$. From the mean performance data in Figure 8, thresholds $(75 \%$ correct points) were calculated. With uncrossed hands, the threshold was $65 \mathrm{msec}$, whereas with crossed hands it was $183 \mathrm{msec}$. These results are consistent with the results from both Yamamoto and Kitazawa (2001a) and Shore et al. (2002) with crossed hands. As Shore et al. reported, even with experienced subjects, crossing the hands results in substantial interference in TOJs.

\section{GENERAL DISCUSSION}

The size of the TOJ bias, as measured by the difference between leading-toward and leading-away trials, appears to vary across Experiments 1, 2, and 3. The largest bias was seen in Experiment 2. In Experiment 2, the subjects made TOJs of ipsilateral stimuli, whereas in Experiments 1 and 3, the judgments were made of bilateral stimuli. In the previous study, bilateral conditions did not result in substantially smaller biases than did ipsilateral conditions. Training and experience in judging temporal order did appear to reduce the bias (Craig \& Busey, 2003). In the present study, the subjects tested in the bilateral conditions had substantially more experience in making TOJs than did the subjects in the ipsilateral condition, which might account for the smaller bias seen in the bilateral conditions. A direct comparison between ipsilateral and bilateral conditions, controlling for the subjects' experience, would be necessary to determine whether the size of the TOJ bias is affected.

In all three experiments, the subjects showed substantial TOJ biases even under conditions in which they were unlikely to have engaged in extensive haptic exploration. The fact that experience in haptic exploration has little effect on the TOJ bias is most clearly seen in Experiment 2. Subjects have considerable experience in manipulating objects or exploring surfaces in such a way that tactile features stimulate adjacent fingers on the same hand as they move across the skin's surface. In this type of exploration, the temporal order and the direction of motion are perfectly correlated. Subjects have virtually no experience in exploring surfaces with their index fingers crossed over their middle fingers, yet the size of the TOJ bias is almost the same in both conditions (Figures 6 and 7). These results and the results with crossed hands offer no support for the explanation that haptic exploration and the close coupling of movement and temporal order is necessary for the TOJ bias.

The conditions under which temporal order is studied are similar to the conditions that produce apparent motion: stimuli presented to two sites with a brief SOA. Tactile apparent motion can be generated not only between two sites on the same side of the body (ipsilaterally), but also between bilateral sites (Sherrick, 1968a, $1968 \mathrm{~b}, 1970)$. It is possible that the TOJ bias effect might be the result of the interaction between the local pattern of motion across the fingerpads and apparent motion generated between the two sites. When the local pattern of motion at one site points directly at the second site, the apparent motion between the two sites is aligned with the local motion. Under those conditions, one might expect maximum interaction between the two types of motion. Altering the alignment between the two types of motion should result in the local pattern's having a smaller effect on apparent motion. When the two directions are orthogonal to each other, the local patterns should not affect apparent motion.

Arguing against a major role for apparent motion is the range of SOAs over which the bias effect is seen: There is a substantial bias at SOAs ranging from 13 to $100 \mathrm{msec}$. Apparent motion depends on SOA. Apparent motion would likely be poor with the pattern durations used in the present experiments at 13-msec SOAs and nonexistent at 200-msec SOAs, yet the TOJ bias is still evident. 
The present results suggest that neither haptic exploration experience nor apparent motion are major factors in the TOJ bias but that the direction of motion, externally defined, is an important factor. The results are consistent with the view that moving patterns produce a shift in attention. With visual stimuli, it has been shown that subjects perceive a moving stimulus ahead of its actual location in space (Balda \& Klein, 1995; Purushothaman, Patel, Bedell, \& Ogmen, 1998). This anticipation of the trajectory of moving stimuli may reflect a spatial shift in attention. If a similar effect were generated by moving tactile patterns, then for a leading-toward stimulus, attention would be moving toward the trailing stimulus. In judging temporal order, subjects report that their attention is drawn from one location to another (Craig \& Busey, 2003). The present results suggest that the extent to which attention shifts from one location to another within an environmental frame may affect the size of the TOJ bias.

The results of these experiments are consistent with the view that subjects use an environmental framework in processing direction of motion. This environmental framework likely results from combining tactile information with information about the position of the hands and fingers in space, a conclusion similar to that reached by Driver and Grossenbacher (1996). Driver and Grossenbacher reached their conclusion on the basis of the fact that the amount of interference between the two index fingers declined as the hands were moved farther apart. In the case of the TOJ bias, changing the distance between hands had no effect on the bias (Craig \& Busey, 2003). Thus, although the TOJ bias is sensitive to changes in spatial position, the effects appear to be limited to orientation in space and are not a function of the distance between the two sites of stimulation.

The information that subjects integrate with the tactile stimulation in developing an environmental framework might be proprioceptive or visual. No attempt was made in these experiments to differentiate these two sources of information by, for example, excluding vision. Such a manipulation might be carried out in future studies.

\section{REFERENCES}

BAlda, M. V., \& Klein, S. A. (1995). Extrapolation or attention shift? Nature, 378, 565-566.

Benedetti, F. (1985). Processing of tactile spatial information with crossed fingers. Journal of Experimental Psychology: Human Perception \& Performance, 11, 517-525.
Benedetti, F. (1986a). Spatial organization of the diplesthetic and nondiplesthetic areas of the fingers. Perception, 15, 285-301.

Benedetti, F. (1986b). Tactile diplopia (diplesthesia) on the human fingers. Perception, 15, 83-91.

BENEDETTI, F. (1988). Localization of tactile stimuli and body parts in space: Two dissociated perceptual experiences revealed by a lack of constancy in the presence of position sense and motor activity. Journal of Experimental Psychology: Human Perception \& Performance, 14, 69-76.

Benedetti, F. (1991). Reorganization of tactile perception following the simulated amputation of one finger. Perception, 20, 687-692.

Bliss, J. C., Katcher, M. H., Rogers, C. H., \& Shepard, R. P. (1970). Optical-to-tactile image conversion for the blind. IEEE Transactions on Man-Machine Systems, MMS-11, 58-64.

Craig, J. C., \& Busey, T. A. (2003). The effect of motion on tactile and visual temporal order judgments. Perception \& Psychophysics, $\mathbf{6 5}$, 81-94.

Driver, J., \& Grossenbacher, P. G. (1996). Multimodal spatial constraints on tactile selective attention. In T. Innui \& J. L. McClelland (Eds.), Attention and performance XVI: Information integration in perception and communication (pp. 209-235). Cambridge, MA: MIT Press.

Oldfield, S. R., \& Phillips, J. R. (1983). The spatial characteristics of tactile form perception. Perception, 12, 615-626.

Parsons, L. M., \& Shimojo, S. (1987). Perceived spatial organization of cutaneous patterns on surfaces of the human body in various positions. Journal of Experimental Psychology: Human Perception \& Performance, 13, 488-504.

Purushothaman, G., Patel, S. S., Bedell, H. E., \& Ogmen, H. (1998). Moving ahead through differential visual latency. Nature, 396, 424.

Rieser, J. J., \& Pick, H. L., JR. (1976). Reference systems and the perception of tactual and haptic orientation. Perception \& Psychophysics, 19, 117-121.

Rinker, M. A., \& Craig, J. C. (1994). The effect of spatial orientation on the perception of moving tactile stimuli. Perception \& Psychophysics, 56, 356-362.

SeKIYAMA, K. (1991). Importance of head axes in perception of cutaneous patterns drawn on vertical body surfaces. Perception \& Psychophysics, 49, 481-492.

Sherrick, C. E. (1968a). Bilateral apparent haptic movement. Perception \& Psychophysics, 4, 159-160.

Sherrick, C. E. (1968b). Studies of apparent tactual movement. In D. R. Kenshalo (Ed.), The skin senses (pp. 331-344). Springfield, IL: Thomas.

Sherrick, C. E. (1970). Temporal ordering of events in haptic space. IEEE Transactions on Man-Machine Systems, MMS-11, 25-28.

Shore, D. I., Spry, E., \& Spence, C. (2002). Confusing the mind by crossing the hands. Cognitive Brain Research, 14, 153-163.

Yamamoto, S., \& Kitazawa, S. (2001a). Reversal of subjective temporal order due to arm crossing. Nature Neuroscience, 4, 759-765.

Yamamoto, S., \& KitaZawa, S. (2001b). Sensations at the tips of invisible tools. Nature Neuroscience, 4, 979-980.

(Manuscript received August 9, 2002 revision accepted for publication December 31, 2002.) 